\title{
Pharmacists subjected to disciplinary action: Characteristics and risk factors
}

DOI:

10.1111/j.2042-7174.2011.00119.x

\section{Document Version}

Accepted author manuscript

Link to publication record in Manchester Research Explorer

\section{Citation for published version (APA):}

Phipps, D. L., Noyce, P. R., Walshe, K., Parker, D., \& Ashcroft, D. M. (2011). Pharmacists subjected to disciplinary action: Characteristics and risk factors. International Journal of Pharmacy Practice, 19(5), 367-373.

https://doi.org/10.1111/j.2042-7174.2011.00119.x

\section{Published in:}

International Journal of Pharmacy Practice

\section{Citing this paper}

Please note that where the full-text provided on Manchester Research Explorer is the Author Accepted Manuscript or Proof version this may differ from the final Published version. If citing, it is advised that you check and use the publisher's definitive version.

\section{General rights}

Copyright and moral rights for the publications made accessible in the Research Explorer are retained by the authors and/or other copyright owners and it is a condition of accessing publications that users recognise and abide by the legal requirements associated with these rights.

\section{Takedown policy}

If you believe that this document breaches copyright please refer to the University of Manchester's Takedown Procedures [http://man.ac.uk/04Y6Bo] or contact uml.scholarlycommunications@manchester.ac.uk providing relevant details, so we can investigate your claim.

\section{OPEN ACCESS}


Title:

Pharmacists subjected to disciplinary action: characteristics and risk factors

\section{Running head}

Disciplined pharmacists

\section{Author names:}

Denham L Phipps $^{\text {a* }}$

Research Associate

[email: Denham.Phipps@manchester.ac.uk]

Peter R Noyce ${ }^{a}$

Professor of Pharmacy Practice

[email: Peter.Noyce@manchester.ac.uk]

Kieran Walshe ${ }^{b}$

Professor of Health Policy and Management

[email: Kieran.Walshe@mbs.ac.uk]

Dianne Parker ${ }^{\mathrm{a}, \mathrm{c}}$

Visiting Professor (University of Manchester); Director (Safety Culture Associates)

[email: Dianne.Parker@safety-culture.co.uk]

Darren M Ashcroft ${ }^{a}$

Professor of Pharmacoepidemiology

[email: Darren.Ashcroft@manchester.ac.uk]

a School of Pharmacy and Pharmaceutical Sciences, University of Manchester, UK

${ }^{b}$ Manchester Business School, University of Manchester, UK

${ }^{\mathrm{c}}$ Safety Culture Associates Ltd, Flintshire, UK 
* Corresponding author: Centre for Innovation in Practice, School of Pharmacy and Pharmaceutical Sciences, Stopford Building, Oxford Road, Manchester M13 9PT, United Kingdom.

Word count: 3,854 (excluding references and tables)

PUBLISHED IN THE INTERNATIONAL JOURNAL OF PHARMACY PRACTICE, VOLUME 19, pp. 367-373, 2011. 


\section{Abstract}

Objective. To establish whether there are any characteristics of pharmacists that predict their likelihood of being subjected to disciplinary action.

Setting. The Royal Pharmaceutical Society of Great Britain's Disciplinary Committee.

Method. One hundred and seventeen pharmacists, all of whom had been referred to the Disciplinary Committee, were matched with a quota sample of 580 pharmacists who had not been subjected to disciplinary action but that matched the disciplined pharmacists on a set of demographic factors (gender; country of residence; year of registration). Frequency analysis and regression analysis were used to compare the two groups of pharmacists in terms of sector of work, ethnicity, age and country of training. Descriptive statistics were also obtained from the disciplined pharmacists to further explore characteristics of disciplinary cases and those pharmacists who undergo them.

Key findings. While a number of characteristics appeared to increase the likelihood of a pharmacist being referred to the disciplinary committee, only one of these - working in a community pharmacy - was statistically significant. Professional misconduct accounted for a greater proportion of referrals than did clinical malpractice, and approximately one-fifth of pharmacists who went before the DC had previously been disciplined by the Society.

Conclusions. This study provides initial evidence of pharmacist characteristics that are associated with an increased risk of being disciplined, based upon the data currently available. It is recommended that follow-up work is carried out using a more extensive dataset in order to confirm the statistical trends identified here.

\section{Keywords}

Risk assessment, Risk factors, Fitness to practice, Disciplinary action, RPSGB, Regulation 


\section{Introduction}

A central theme within healthcare regulation is the desire to minimise the risk posed by particular practitioners or types of practice [1]. The Department of Health (DoH) [2] has recommended that healthcare practitioners should be subjected to risk profiling in order to ensure that regulatory efforts are targeted appropriately. The DoH suggests a range of criteria that could be used to distinguish "high risk" from "low risk" practitioners; for example the time since registration, the type of work being carried out, and previous performance (including both general performance ratings and whether or not the practitioner has previously faced sanctions). The Royal Pharmaceutical Society of Great Britain (RPSGB) [3] aspires to use such information to tailor revalidation processes to the relative risk of pharmacists; high risk practitioners will be expected to undergo a more intensive revalidation schedule than those who are designated as low risk.

There is some evidence to suggest that certain characteristics of a practitioner can predict his or her likelihood of being the subject of disciplinary action. Amongst nurses and physicians in the US, both age and the time since first registration have been found to predict the likelihood of being disciplined however, the direction of the linear relationship varies between studies [4-7]. Amongst British and American physicians, those working in general practice, surgery, obstetrics and gynaecology, and psychiatry are most likely to be referred to a disciplinary panel [5,7-9]. Male practitioners, non-white practitioners, practitioners from certain socioeconomic backgrounds and practitioners trained overseas have also been found to be at increased risk of being disciplined [4-6, 8-10]. Finally, a study of UK general practitioners has found that those working alone are most likely to be disciplined [11], while in the UK and the US, physicians who performed poorly at medical school, or who received sanctions for professional misconduct during their training, appear more likely to be the subject of disciplinary action later [12-14]. There are, as yet, no such studies involving pharmacists; however, there is some evidence from qualitative research that advanced and specialist practice brings with it particular types of risk [15]. Alternatively, it could be the case 
that in general terms, pharmacists working in these areas are less risky due to their having greater expertise in the fields in which they are working [16].

These studies suggest the possibility of healthcare practitioners, including pharmacists, being assessed by "risk scoring" specific factors such as demographic variables and employment status [17]. However, in the context of pharmacy practice, it is not clear what the influence of these factors on risk is, if indeed they are of any influence at all. The aim of the present study is to explore the relationship between pharmacist characteristics and their risk of being disciplined. 


\section{Materials and Methods}

Ethical approval for the study was granted by the University of Manchester Senate Committee. The study used a case-control design, in which pharmacists who had undergone a disciplinary hearing were compared with matched control cases (pharmacists who had not undergone a hearing). In order to identify pharmacists who had undergone a hearing, the researchers used the repository of fitness to practice records published on the RPSGB's website. These records provide particulars of cases that have been heard by the Society's Disciplinary Committee (DC) following an allegation of poor performance or misconduct. A case will be referred to the $D C$ if there is believed to be an impairment of fitness to practice that is not due to health impairment and either: (a) it cannot be resolved by words of advice from the Society (for example, because of the gravity of the offence or because the registrant has failed to comply with a previously agreed undertaking); (b) the participant does not accept that his or her fitness to practice is impaired [18].

The criteria for including a DC case in the sampling frame was that it was received by the Society after 1 April 2007 (on which date a new disciplinary framework was introduced) and that it was a new disciplinary case, rather than an application for restoration to the register following a period of suspension. This ensured that all cases had been reviewed against the same standards of practice. For each disciplined pharmacist, five non-disciplined pharmacists, who were matched in terms of gender, country of residence and year of registration, were chosen at random from the RPSGB registration database. The choice of five control cases for each case in the sample provides a sample size that is sufficiently large for a multivariate analysis [19], whilst being cautious to avoid masking any effects because of too high a ratio of control cases. For all pharmacists in the sample, demographic details (date of birth; ethnicity; sector; whether qualified in the UK or abroad; whether qualified as a prescriber) were recorded. For pharmacists in the disciplined group, details of the case (source of the complaint; nature of the complaint; outcome of any hearing; whether the pharmacist had previously been the subject of disciplinary action) were also recorded. The researchers obtained their data from public-domain information published on the RPSGB website 
and from the RPSGB's membership database. Data from the latter, due to its not being in the public domain, was compiled by RPSGB staff and anonymised at source before transmission to the researchers. 
Data analysis

All data were entered into SPSS Version 15.0 and anonymised prior to analysis. A logistic regression was carried out with the pharmacist's sector, country of qualification, ethnicity and age as predictor variables and allocation to the disciplined or control (non-disciplined) group as the dependent variable. As a preliminary to the logistic regression, a log-linear analysis was performed in order to compare the effects of different combinations of the predictors. Due to low cell frequencies within the sector, ethnicity and country of qualification variables, these were recoded prior to analysis (Sector: hospital; community; other. Ethnicity: white; non-white. Country: UK; non-UK). Registration as a prescriber was removed from the predictors due to the outcome groups being perfectly separated on this variable, leading to large values of standard error. Because the log-linear analysis uses categorical data, age was categorised for the purpose of this particular analysis. The categories, which are shown in Table 1, were chosen arbitrarily to provide an even spread of cases in each in each category. In the logistic regression, age was used in its continuous form. Prior to each run of the regression analysis, cases with missing data on the independent variable(s) were removed using listwise deletion. 


\section{Results}

How many pharmacists were included in the study?

One hundred and seventeen pharmacists who met the criteria for inclusion in the study were identified in the online repository as of December 2009 (when data extraction was carried out); all of these were included in the study. Using the criteria defined earlier, an additional sample of 580 non-disciplined pharmacists was obtained from the RPSGB's membership database, comprising the control group. Hence the total sample comprised 697 pharmacists. Details of this sample are shown in Table 1.

\section{INSERT TABLE 1 HERE}

Do pharmacists' demographic characteristics predict the likelihood of their being referred to the Disciplinary Committee?

From the log-linear analysis, the most parsimonious model was identified through stepwise selection by deletion of effects. This model contained only sector as a predictor. The likelihood ratio $x^{2}(57)=30.588, p=0.998$, indicating a good fit between observed frequencies and the expected frequencies generated by the model. The proportion of community pharmacists in the disciplined group is greater than that in the control group. However, inspection of the descriptive statistics in Table 1 indicates that the modal sector in both groups is community pharmacy.

The regression analysis found a good model fit on the basis of all four predictors $\left[x^{2}(5, N=514)=12.591, p=0.028\right]$, indicating that the predictors, as a set, reliably distinguished between disciplined and non-disciplined pharmacists. However, the variance in outcome accounted for by the model is rather modest [Cox \& Snell R-Square $=0.024$; Nagelkerke R-Square $=$ $0.043]$.

Table 2 shows the Exp-b odds ratios and their 95\% confidence intervals for each of the four predictors. According to the Wald criterion, only the pharmacist's sector has a statistically significant effect on the classification. Specifically, pharmacists working in community pharmacy were at increased 
risk of being disciplined when compared to pharmacists in non patient-facing roles. In addition, a relatively high but statistically non-significant odds ratio was obtained when comparing community pharmacists to hospital pharmacists (odds ratio $=2.302, p=0.088$ ). With the exception of the pharmacist's age (which is very highly correlated with the matching variable of years since registration: Pearson's $r=0.929, N=694, p<0.001$ ), the other predictors also show odds ratios greater than 1 , although none of these reach statistical significance. The univariate odds ratios for overseas training and non-White ethnicity come close to being statistically significant (with $p$ values of 0.068 and 0.094 respectively), although in the multivariate model these predictors are no longer close to significance ( $p$ values increase to 0.489 and 0.570 respectively).

\section{INSERT TABLE 2 HERE}

What are the other characteristics of disciplinary referrals?

Table 3 provides further descriptive statistics about the disciplined pharmacists and the circumstances surrounding their being reported to the RPSGB. As the table shows, misconduct (violation of standards; dishonesty; behaviour) is cited more frequently than clinical malpractice (dispensing errors; controlled drug errors). It should be noted, though, that in 28 of the cases, two or more reasons were given for referral. That said, the same pattern is observed even amongst those pharmacists who were reported against one category only; indeed, the difference between misconduct and clinical malpractice frequencies becomes even larger (72 versus 16 citations respectively).

The most frequent source of complaints is from someone who is in a position of oversight of the pharmacist - for example, the employer, the primary care organisation or a Society inspector. However, there are a range of other routes by which a pharmacist can be brought to the attention of the Society: approximately seventeen percent of referrals are triggered by the police, ten percent by a member of the public, and yet others by peer- or self-referral. 


\section{INSERT TABLE 3 HERE}

\section{Discussion}

The findings of the current study indicate patterns in the demographic characteristics of pharmacists who are referred to the Society's Disciplinary Committee. However, the factors that predict a pharmacist being disciplined appear to be limited to the pharmacist's sector; specifically, whether the pharmacist is working in community pharmacy, and possibly also whether the pharmacist is working in a patient-facing role. In addition, there is a noticeable but statistically non-significant effect of the pharmacist's qualification and his or her ethnicity.

The study also identified patterns in the circumstances surrounding pharmacists being referred to the Society's Disciplinary committee. Of those pharmacists who were referred to the DC, approximately one fifth had previously been the subject of disciplinary action by the Society. Also, while referrals are triggered by a variety of sources, the employer or primary care organisation is the most frequent source, although a sizeable proportion of referrals also came from the police, RPSGB inspectors and members of the public. Of the different reasons cited for referral to the Society, conduct concerns appear more frequently than clinical malpractice.

While the study has been able to identify some patterns and relationships within the data, a few limitations need to be considered, aside from the generally low strength of prediction provided by the regression model. Firstly, it should be noted that, as alluded to previously, the Disciplinary Committee represents the end stage of a process that begins with a review of the case by the Investigating Committee (IC). The most serious cases will be referred from the IC to either the DC or the Health Committee $(\mathrm{HC})$, but a substantial proportion of the cases are dealt with by the IC itself. The RPSGB [20] reports that, between 1 April 2008 and 31 March 2009, the IC reviewed 636 cases, of which only 77 were referred to the DC; a similar proportion were also referred during the previous year [21]. Hence, the data used in this study represents a small subset of the population of investigated pharmacists. 
Secondly, it should be noted that the study involves retrospective analysis of records that were not initially designed to elucidate risk factors. As has been indicated in the foregoing text, some data was not fully available to the researchers. Furthermore, it is possible that other items of information, not currently collected from pharmacy practitioners on a routine basis, could be used as risk factors. Examples include: hours worked per week; length of time in current job; whether the practitioner has recently changed sectors; whether he or she is working as an advanced or specialist practitioner, and whether he or she is working alone. In the context of tailoring revalidation regimes, indicators such as these also have the advantage of being more transparent, and possibly more defensible, than are demographic factors such as socio-economic status and ethnicity.

Finally, the time window represented by the cases in this study is quite limited. It is insightful to view the findings in the context of the demographic trends identified by Seston \& Hassell [22]. They note that between 2002 and 2009, amongst other demographic changes, there has been an increase in the proportion of members who are non-white and, amongst those pharmacists who were trained outside the UK, an increase in the proportion that are accredited via the European route. In addition, there has been an increase in the number of pharmacists who are qualified as independent prescribers. Given the odds ratios reported earlier, it is possible that, as more data accumulates from disciplinary hearings, other statistically significant risk factors could emerge.

The finding that community pharmacists make up a larger proportion of Disciplinary Committee referrals than do hospital pharmacists could be an artefact of there being greater numbers of the former; alternatively, it may reflect a genuine difference between the two sectors in terms of risk. For example, the greater prevalence of sole practitioners in community pharmacy could make it intrinsically riskier than hospital pharmacy, in which the pharmacist is more likely to be engaged in collaborative work. However, by way of a caveat this finding should be put into the context of differences between the two sectors. Anecdotal and empirical evidence suggests that 
some healthcare organisations are more inclined or able than others to deal with disciplinary matters "in-house" without referral to an external body [16]. In the United Kingdom, many hospital pharmacists are employed by NHS trusts, which are likely to have their own disciplinary processes. This may also be the case in the larger community pharmacy chains, where a hierarchical "command structure" is often present. However the smaller community pharmacies (particularly those where the pharmacist is also the superintendent and/or sole proprietor) may not have a structure in place for addressing fitness to practice concerns, and hence those who wish to raise such concerns would need to involve the Society. Of the 89 community pharmacists who were in the disciplined group in the current study, details about the type of pharmacy were available only for 30 . Eleven of these were in independent pharmacies, 8 were in a small or medium chain, a further 8 in a large chain, and 3 in a supermarket. In addition, as indicated in the previous section, pharmacy owners appear relatively frequently amongst disciplined pharmacists; however, there does not appear to be any particular type of disciplinary offence that is more attributable to these pharmacists than to others. Interestingly, data recently released by the National Clinical Assessment Service (NCAS) [23] indicates that since 1 April 2009 (when NCAS began to take referrals of pharmacists with performance concerns), referrals of community pharmacists have outnumbered those from hospitals. This echoes the findings of the current study. However, the authors of the NCAS report have emphasised that their data is from the first 14 months of operation only, and that any trends in the data would need to be followed for a longer period of time before any definitive conclusions can be made.

The findings of the current study are, in general, broadly consistent with other studies. For example, amongst healthcare professionals, age, ethnicity, occupation and country of training have been found to predict the likelihood of being the subject of disciplinary action [9]. With regard to pharmacists specifically, a previous study [24] has found that a disproportionate number of ethnic minority pharmacists were being referred to the RPSGB's Statutory Committee (the precursor to the IC and DC). The reason for these patterns, however, is not clear. Are ethnic minority pharmacists or those trained 
outside the UK inherently more risky, or are there other factors at play? One might surmise, for example, that while some pharmacists are able to resolve problems informally without recourse to any disciplinary framework [25], it may be less easy for a pharmacist to do so if there exists a cultural or linguistic barrier between the pharmacist and colleagues or patients [16]. It appears apposite to explore the issues surrounding non-UK trained pharmacists in future research [26]. Incidentally, as suggested earlier, the finding that age was unrelated to the risk of being disciplined may represent either a genuine effect in the population or an artefact of the sampling strategy used for this study, in which pharmacists in both groups were matched on the basis of their length of time in the profession. The data from NCAS pharmacist referrals did not find any particular trends in terms of age although, as with the current study, that data is drawn from a limited sample (as discussed earlier) [23].

That one-fifth of disciplined pharmacists have previously been referred to the Society is particularly noteworthy given that, in any one year, the proportion of pharmacists on the entire RPSGB database who is disciplined is approximately $1 \%$ [20-21]. It is possible that previous disciplinary action may indeed be a predictor of future disciplinary action, although no data from the case controls was available for the current study, so this hypothesis could not be tested. Also, the data used in the current study pertains only to previous disciplinary action by the RPSGB; hence, it may be worth collecting data in the future about disciplinary action by other professional bodies, by the School in which a pharmacist trained, or his or her being the subject of criminal or civil action.

At the time of data collection, the role of the pharmacist at the time of the incident was not routinely recorded either. The frequencies cited for pharmacist roles have been collated from the information available in DC determinations or on the membership database, and may not pertain to the pharmacist's role at the time of the incident. These caveats in mind, it would appear that the most frequently occurring role is proprietor or owner, with employed (non-manager) pharmacists and locum pharmacists the next most frequent roles. It might be surmised that the former are more likely to work 
alone, or that (unlike in the case of employed pharmacists) there is no line management to whom practice concerns can be addressed, and hence Society referral is the only option available. Similarly, it is possible that the reason for the pharmacist being reported in the first place may be different from that given on referral to the DC; it appears from a review of the publicly available DC cases that in some of them, the particulars as described in the determination are not consistent with the reason given in the database. By way of illustration, examples of the two types of complaint are shown in Table 4.

As a general observation, it is noted that prior to the introduction of a new framework in 2007, clinical competence lay outside the remit of the RPSGB's disciplinary procedures, which instead focused on professional misconduct. This may be a "legacy" factor that accounts for the latter making up a greater number of referrals to the current disciplinary committee. Again, it is interesting to note by way of comparison that pharmacist referrals to NCAS (in contrast to referrals of doctors and dentists) appear to happen more frequently for governance, misconduct or health concerns than for clinical concerns [23]. It is also notable that previous studies identify colleagues and patients as the most frequent source of referrals of healthcare professionals [27]. The data from the current study indicate that, while referrals to the Society that result in a DC hearing come from a variety of sources (including colleagues and customers), the employer or primary care organisation is the most frequent source, followed by the police, Society inspectors and members of the public. Whether there is a need for changes to policies, procedures or reporting mechanisms - either within the regulatory body or within pharmacy employers - to reflect the remit of the new framework may be an issue that merits further consideration.

\section{INSERT TABLE 4 HERE}

In summary, it is difficult to draw any clear conclusions with the limited data available. However, a number of issues are identified from the analysis, 
which are worth exploring in future work as more data from disciplinary hearings accumulate.

\section{Conclusion}

There is the potential for risk factors to be identified from the disciplinary records held by pharmacists' regulatory bodies. The current study has used currently available data from the RPSGB to identify issues of concern, and indicated areas in which further work would be useful, either in terms of data recording or in following up some of the issues identified. Future research should focus on as wide as a range of indicators as possible; these might usefully include demographic data, work history, and current employment characteristics.

\section{Acknowledgements}

This work was commissioned by the Royal Pharmaceutical Society of Great Britain and funded by the Department of Health. The authors would like to thank the RPSGB Revalidation Research Steering Committee for their comments on an earlier version of this paper. 


\section{References}

1. Phipps $D$ et al. What factors should you focus on to provide meaningful risk assessments? Pharmceut J 2010; 284: 312.

2. Department of Health. The Regulation of the Non-Medical Healthcare Professions. Leeds: DH, 2006.

3. Royal Pharmaceutical Society of Great Briatin. A Draft Model for Revalidation in Pharmacy: report to the Department of Health. London: RPSGB, 2009

4. Clay SW, Conatser RR. Characteristics of physicians disciplined by the State Medical Board of Ohio. J Am Osteopath Assoc 2003; 103: 81-88.

5. Morrison J, Wickersham P. Physicians disciplined by a state medical board. J Am Med Assoc 1998; 279: 1889-1893.

6. Green $A$ et al. Are you at risk for disciplinary action? Am J Nursing 1995; 95: 36-42.

7. Dehlendorf CE, Wolfe SM. Physicians disciplined for sex-related offenses. J Am Med Assoc 1998; 279: 1883-1888.

8. Khaliq AA et al. Disciplinary action against physicians: Who is likely to get disciplined? Am J Med 2005; 118: 773-777.

9. National Patient Safety Agency. NCAS Casework: the first eight years. London: NPSA, 2009.

10. Yates J, James D. Risk factors at medical school for subsequent professional misconduct: multicentre case-control study. $\mathrm{Br} \mathrm{Med} J$ 2010; 340: c2040.

11. Watts S. Solo GPs face more GMC hearings. http://news.bbc.co.uk/1/hi/programmes/newsnight/8163826.stm (accessed 22nd July 2009).

12. Hickson GB et al. A complementary approach to promoting professionalism: identifying, measuring and addressing unprofessional behaviours. Acad Med 2007; 82: 1040-1048.

13. Papadakis MA et al. Disciplinary action by medical boards and prior behavior in medical school. New Engl J Med 2005; 353: 2673-2682.

14. Papadakis MA et al. Performance during medicine residency training and subsequent disciplinary action by state licensing boards. Ann Intern Med 2008; 148: 869-876. 
15. Carthey $\mathrm{J}$ et al. Development of a risk-based approach for the identification of advanced pharmacy practice. Report to the Royal Pharmaceutical Society of Great Britain, 2007.

16. Phipps DL et al. Risk assessment in pharmacy practice. Report to the Royal Pharmaceutical Society of Great Britain, 2010.

17. Phipps DL et al. Risk-based regulation: what are the implications for pharmacy practice? Health Risk Soc 2011 (in press).

18. Her Majesty's Stationery Office. Royal Pharmaceutical Society of Great Britain (Fitness to Practice and Disqualification etc. Rules) Order of Council. SI 2007/442. London: HMSO, 2007.

19. Tabachnick BG, Fidell LS. Using Multivariate Statistics, $4^{\text {th }}$ edn. Boston: Allyn \& Bacon, 2001.

20. Royal Pharmaceutical Society of Great Britain. Fitness to Practice: annual report 2008. London: RPSGB, 2009.

21. Royal Pharmaceutical Society of Great Britain. Fitness to Practice: annual report 2007. London: RPSGB, 2008.

22. Seston E, Hassell K. Workforce update - joiners, leavers and practising and non-practising pharmacists on the 2009 register. Pharm J 2010; 284: 80-82.

23. National Clinical Assessment Service. A Review of the Extension of the National Clinical Assessment Service to Pharmacists. London: NCAS, 2010.

24. Hassell K. A study of Statutory Committee charges and ethnic origin. Pharmaceut J 1996; 257: R43.

25. Phipps DL et al. Medication safety in community pharmacy: a qualitative study of the sociotechnical context. BMC Health Serv Res 2009; 9: 158.

26. Ziaei $Z$ et al. Experiences of European trained pharmacists in Great Britain. Int J Pharm Pract 2010; 18 (Supplement 1): 9-10.

27. Cox SJ, Holden JD. Presentation and outcome of clinical poor performance in one health district over a 5-year period: 2002-2007. $\mathrm{Br}$ J Gen Pract 2009; 9: 344-348. 


\section{Tables and figures}

Table 1: Demographic characteristics of the sample

Table 2: Results from the regression of group allocation onto the demographic predictors

Table 3: Characteristics of disciplinary cases

Table 4: Types of disciplinary case 
Table 1 Demographic characteristics of the sample

\begin{tabular}{|c|c|c|c|}
\hline & \multicolumn{2}{|c|}{ Group } \\
\hline & & Disciplined & Control \\
\hline Total N & & 117 & 580 \\
\hline \multirow[t]{2}{*}{ Gender* } & Male & $92(78.6 \%)$ & $455(78.4 \%)$ \\
\hline & Female & $25(21.4 \%)$ & $125(21.6 \%)$ \\
\hline \multirow[t]{4}{*}{ Country ${ }^{*}$} & England & $100(85.5 \%)$ & $495(85.3 \%)$ \\
\hline & Scotland & $8(6.8 \%)$ & $40(6.9 \%)$ \\
\hline & Wales & $4(3.4 \%)$ & $20(3.4 \%)$ \\
\hline & Other & $5(4.3 \%)$ & $25(4.3 \%)$ \\
\hline \multirow[t]{10}{*}{ Sector } & Hospital & $8(6.8 \%)$ & $69(11.9 \%)$ \\
\hline & Community & $89(76.1 \%)$ & $379(65.3 \%)$ \\
\hline & РCT & $2(1.7 \%)$ & None \\
\hline & Industry & $1(0.9 \%)$ & $27(4.7 \%)$ \\
\hline & Academia & $2(1.7 \%)$ & $6(1.0 \%)$ \\
\hline & Wholesale & None & $1(0.2 \%)$ \\
\hline & Other pharmaceutical & None & $12(2.1 \%)$ \\
\hline & Non-paid & None & $3(0.5 \%)$ \\
\hline & Non-pharmaceutical & None & $14(2.4 \%)$ \\
\hline & No information available & $15(12.8 \%)$ & 69 (11.9\%) \\
\hline
\end{tabular}

$\begin{array}{cll}\text { Qualification }^{\text {Adjudication } / \text { OSPAP }}{ }^{+} & 7(6.0 \%) & 24(4.1 \%) \\ \text { Reciprocal }^{+} & 5(4.3 \%) & 9(1.6 \%) \\ \text { European } & 4(3.4 \%) & 15(2.6 \%) \\ \text { UK } & 101(86.3 \%) & 532(91.7 \%)\end{array}$

Registered prescriber?

Yes None

$14(2.4 \%)$

No $117(100 \%)$ $566(97.6 \%)$ 
Table 1 Demographic characteristics of the sample (continued)

\begin{tabular}{|c|c|c|c|}
\hline & \multicolumn{2}{|c|}{ Group } \\
\hline & & Disciplined & Control \\
\hline \multirow[t]{17}{*}{ Ethnicity } & White British & $38(32.5 \%)$ & $295(50.9 \%)$ \\
\hline & White Irish & $1(0.9 \%)$ & $9(1.6 \%)$ \\
\hline & White Other & $8(6.8 \%)$ & $22(3.8 \%)$ \\
\hline & Black Caribbean & None & $2(0.3 \%)$ \\
\hline & Black African & $6(5.1 \%)$ & $15(2.6 \%)$ \\
\hline & White and Black African & None & $1(0.2 \%)$ \\
\hline & White and Asian & None & $1(0.2 \%)$ \\
\hline & Mixed Other & None & $3(0.5 \%)$ \\
\hline & Indian & $19(16.2 \%)$ & $96(16.6 \%)$ \\
\hline & Pakistani & $7(6.0 \%)$ & $26(4.5 \%)$ \\
\hline & Bangladeshi & None & $3(0.5 \%)$ \\
\hline & Asian Other & $2(1.7 \%)$ & $7(1.2 \%)$ \\
\hline & Chinese & $3(2.6 \%)$ & $14(2.4 \%)$ \\
\hline & Other ethnic group & None & $4(0.7 \%)$ \\
\hline & Declined to answer & $1(0.9 \%)$ & $6(1.0 \%)$ \\
\hline & Did not return census form & $29(24.8 \%)$ & $76(13.1 \%)$ \\
\hline & No information available & $3(2.6 \%)$ & None \\
\hline \multirow[t]{7}{*}{ Age } & $25-34$ years & $19(16.2 \%)$ & $108(18.6 \%)$ \\
\hline & $35-44$ years & $20(17.1 \%)$ & $100(17.2 \%)$ \\
\hline & $45-54$ years & $36(30.8 \%)$ & $184(31.7 \%)$ \\
\hline & $55-64$ years & $33(28.2 \%)$ & $165(28.4 \%)$ \\
\hline & $65+$ years & $6(5.1 \%)$ & $23(4.0 \%)$ \\
\hline & No information available & $3(2.6 \%)$ & None \\
\hline & Mean, standard deviation & $48.7,11.3$ & $48.0,11.4$ \\
\hline \multicolumn{2}{|c|}{ Year of registration (median, range) ${ }^{*}$} & 1984,59 & 1984,59 \\
\hline \multicolumn{2}{|c|}{ Year of birth (median, range) } & 1959,51 & 1960,64 \\
\hline
\end{tabular}

Note: * indicates a variable that was used to match participants in either group. ${ }^{+}$Adjudication / OSPAP qualifications are those that were obtained outside the EU and subsequently 
validate in the UK via a post-qualification diploma. Reciprocal qualifications are those that are obtained outside the UK but considered equivalent by virtue of a reciprocal agreement between the respective countries. Historically this included Australia and New Zealand, but now applies to Northern Ireland only. 
Table 2 Results from the regression of group allocation onto the demographic predictors

\begin{tabular}{|c|c|c|}
\hline Univariat & & Multivariate \\
\hline $95 \% \mathrm{Cl}$ & $p$ & OR \\
\hline
\end{tabular}

Sector:

Hospital (vs Other) $\quad \mathbf{1 . 4 6 1} \quad 0.454,4.699 \quad 0.525 \quad 1.531 \quad 0.347,6.745 \quad 0.574$

Community (vs Other)2.959* 1.156, $7.570 \quad 0.024 \quad \mathbf{3 . 5 2 3}^{*} 1.056,11.7530 .041$

Qualification:

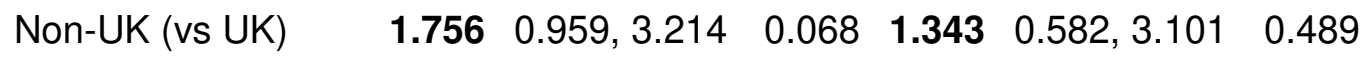

Ethnicity:

Non-White (vs White) $1.492 \quad 0.934,2.384 \quad 0.094 \quad 1.166 \quad 0.685,1.985 \quad 0.570$

\begin{tabular}{lllllll} 
Age & $\mathbf{1 . 0 0 5}$ & $0.988,1.023$ & 0.572 & 1.019 & $0.995,1.044$ & 0.117 \\
\hline
\end{tabular}

Key: OR: Odds Ratio. ${ }^{*}$ Wald statistic $p<0.05$. For the Sector predictor, $\mathrm{df}=2$. For the other predictors, $\mathrm{df}=1$. 
Table 3 Characteristics of disciplinary cases

Frequency Percentage

of cases of cases

Reason for investigation:

Violation of professional / legal standards 34

38.2

Dishonesty

$33 \quad 37.1$

Controlled drug errors

$9 \quad 10.1$

Dispensing errors

7

6.0

Behaviour towards customers and colleagues 5

5.6

Health problem

1

1.1

More than one reason

28

24.0

Subject of previous disciplinary action by the Society?

Yes

No

Source of complaint:

Employer

Primary care organisation

Police

Inspector / Society

Member of public

CFSMS $^{\circ}$

Self-referral

Another Society member

Another healthcare professional

Co-worker

Other enforcement agency
22

95

18.8

81.2
23

20

20

15

19.7

17.1

17.1

12.8

12

10.3

10

8.5

6

5.1

4

3.4

4

3.4

2

1.7

1

\section{9}


Table 3 Characteristics of disciplinary cases (continued)

\begin{tabular}{|c|c|c|}
\hline & $\begin{array}{l}\text { Frequency } \\
\text { of cases }\end{array}$ & $\begin{array}{l}\text { Percentage } \\
\text { of cases }\end{array}$ \\
\hline \multicolumn{3}{|l|}{ Pharmacist's role:* } \\
\hline Proprietor / owner & 18 & 15.4 \\
\hline Employed pharmacist & 11 & 9.4 \\
\hline Locum pharmacist & 10 & 8.5 \\
\hline Manager & 8 & 6.8 \\
\hline Superintendent & 6 & 5.1 \\
\hline Other & 4 & 3.4 \\
\hline Unknown & 60 & 51.3 \\
\hline
\end{tabular}

Notes: * This data was collected from a variety of sources, some of which may not relate to the time at which the incident occurred. ${ }^{\circ}$ CFSMS: Counter Fraud and Security Management Service. 
Table 4 Types of disciplinary case

Type

Violation of professional / legal standards

Dishonesty

Behaviour towards customers and colleagues

Controlled drug errors

Dispensing errors: Strength and dosage

Incorrect medication

Labelling

Out of date supply

Conviction / caution Health problem:

Drugs

Alcohol

\section{Example}

Taking controlled drugs from patient returns and dispensary stock for own use Committing tax fraud

Inappropriate or dismissive remarks made to a colleague about a work matter Failing to properly dispose of patient-returned controlled drugs

Repeatedly dispensing a different strength of medication to that described on the prescription and/or label

Repeatedly dispensing different medicines to those described on the prescription and/or label

Dispensing methadone mixture against prescriptions for dexamphetamine elixir Allowing out-of-date medicines to be mixed with in-date stock and dispensed Being convicted of driving under the influence of alcohol Having a drug dependency Having an alcohol dependency 